\title{
Impact of Adverse Reactions on Adherence To Treatment For Mdr - Tb Under Pmdt in A Tertiary Care Teaching Hospital
}

\author{
Dr.Keerthivasan Sivanmani M.D(Respiratory medicine $)^{1}$ \\ ${ }^{I}$ Department Of TB \& Chest Diseases, Coimbatore Medical College,The Tamilnadu Dr.MGR Medical \\ University, India.
}

\begin{abstract}
Aim: To study the adverse reactions for second line drugs in the treatment of MDR TB and its impact on adherence to the treatment

Settings and Design: Department of TB \& Chest diseases, Coimbatore medical college Hospital

Prospective study

Period of study: Jan 2014 to Dec 2014

Methods and Material: Adverse events in 109 patients of MDR-TB started on second line drugs in our tertiary care centre were observed from January2014 to December 2014. Lists of 11 adverse reactions were selected and the patients were assessed for adverse events during the $1^{\text {st }}$ week of inpatient treatment and at the end of $1^{\text {st }}$ month of treatment.

Results:It was observed that among the 109 patients enrolled for treatment 46 patients (42\%) had any one adverse drug reaction event. Among the MDR patients change of regimen was needed in only $5 \%$ of cases due to adverse event which is very low. Only $2.7 \%$ of cases discontinued treatment due to adverse reaction. This study shows that most of the adverse reactions are manageable with the standard management strategies and the default rates are very low due to adverse events.

Conclusion: In resource limited settings as in India, proper development of treatment strategy, protocols to manage the adverse reactions and proper follow up as in PMDT model to treat MDR - TB leads to high percentage of continuation of treatment without interruptions.
\end{abstract}

Keywords: $m d r-t b$, genexpert, line probe assay

\section{Introduction}

The emergence of resistance to drugs used to treat tuberculosis particularly multi drug resistant TB has become a significant public health problem in India and an obstacle to effective TB control. In India the available information from several drug resistance surveillance studies conducted in the past suggest that the rate of MDR-TB is relatively low in India ${ }^{1}$. However this translates into a large absolute number of cases. Specific measures are taken within the Revised National Tuberculosis control programme (RNTCP) to address the MDR - TB problem (i.e.) programmatic management of Drug Resistant Tuberculosis (PMDT). Adherence to the treatment is a critical factor in the management of MDR-TB ${ }^{2}$ and the adverse events associated with second line drugs $^{3}$ could have a serious impact on adherence. This report presents the adverse drug events in patients started on cat IV in a tertiary care medical college and its impact on adherence to the treatment

\section{Methods And Materials}

The study population included was the patients diagnosed as MDR -TB between Jan-2014 to Dec-2014 in our institute. The diagnosis of MDR-TB was based on the results of either Gene Xpert or Line Probe Assay. The samples for Line Probe Assay were sent to Intermediate reference laboratory (IRL) at Chennai and for Gene Xpert the facility in our institute is utilized. Criteria C was followed for Drug sensitivity Testing (DST) as per RNTCP guideline. Patients who are defaulters, Relapse and failures in previously treated tuberculosis, contact of MDR-TB and newly diagnosed tuberculosis with HIV were subjected to DST. MDR TB was diagnosed either if patient showed resistance to both INH \& Rifampycin in LPA or Rifampycin alone in Gene Xpert ${ }^{1}$.

Once the diagnosis was made patients were admitted in out DOTS - PLUS Ward. Pre-treatment evaluation with complete blood count, RFT, LFT, S. electrolytes, Thyroid function test, ECG was done. After pre-treatment evaluation patient were started on Cat IV of RNTCP which includes Kanamycin (KM), Levofloxacin (Levo), Ethionamide (ETO), Cycloserine (CS), Pyrazinamide (Z), and Ethambutol. PAS, Moxifloxacin (MFC), Capreomycin (CM) were kept as reserve/substitute drugs. Patients were observed in the ward for 7 days for any adverse reactions after which the patients were discharged.

Lists of 11 (Table.1) adverse reactions were selected and the patients were assessed for adverse events during the 1 week of inpatient treatment and at the end of $1^{\text {st }}$ month of treatment. 


\section{Results}

A total of 109 patients who were started on MDR treatment between Jan 2014 to Dec 2014 were included in the study. Among the 109 patients 89 were males and 20 females (fig.1). Age wise distribution of the patients showed majority in the age group between 18-45 and above 45. In DST, 54 patients were tested by Gene Xpert (50\%), 53 by LPA (49\%) \& one patient by L J medium (fig. 2). Relapse (50\%) was the most common indication for DST followed by failure (20\%) and defaulters (20\%), and HIV (7\%) (fig.3). 4 patients died during the treatment. 8 patients defaulted during the treatment. Only 3 patients $(2.7 \%)$ defaulted due to adverse events while in others the cause for default was alcoholism \& alternative system of medicine. $5 \%$ of patients needed regimen change due to adverse reaction and the most common adverse event for regimen change was hearing loss. In Table. 1 the frequency of adverse events were enumerated. The most common were GI Symptoms (39\%), giddiness (13\%), Hearing loss (4\%), Arthralgia (3\%). The most life threatening adverse event was Steven Johnson syndrome in a patient who was HIV reactive.

\section{Discussion}

The management of MDR - TB is a complex process and involves various factors which play an obstacle in the completion of long duration of treatment of 24 months. Although studies from United States and Hong Kong (non- resource limited settings) shows high rate of discontinuation of drugs of $30 \%$ and $19 \%$ respectively due to adverse events ${ }^{4,5}$, our study (resource limited settings) showed only $2.7 \%$ discontinuation rate due to adverse events. This Study shows that adverse reactions do not play a major role in the non

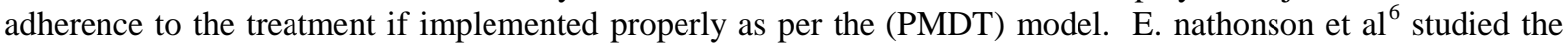
adverse reaction in MDR patients from multiple centres across the world and showed only $2.1 \%$ of patients stopped treatment due to adverse events which correlated with our study. Also the GI symptom was the most common adverse reactions observed by E. nathonson et al as well as in our study which requires reassurance and symptomatic management alone. PMDT model of initiation of MDR - TB regimen as an inpatient treatment for 7 days plays a critical role in managing the adverse reaction particularly the GI symptoms and other allergic reactions. Also in the PMDT model adverse reactions for each specific drug has been outlined and standard protocols were developed to manage it. Training was given to manage those adverse reactions up to the community health workers level. This leads to early detection of adverse events and appropriate intervention as early as possible.

\section{Conclusion}

In resource limited settings as in India, proper development of treatment strategy, protocols to manage the adverse reactions and proper follow up as in PMDT model to treat MDR - TB leads to high percentage of continuation of treatment without interruptions. As in our study only $2.7 \%$ of patients discontinued treatment due to adverse reaction and in only $5 \%$ of patients a change in regimen was made to combat the adverse reactions. In spite of limitation in this study like sample size, single centre study, it shows that in $97 \%$ of patients it was possible to avoid discontinuation of drugs due to adverse reactions.

\begin{tabular}{|l|l|l|}
\hline \multicolumn{2}{|c|}{ Figures and table } \\
\hline \multicolumn{3}{|c|}{} \\
\hline S.NO & ADVERSE REACTIONS & NO.OF PATIENTS \\
\hline 1 & Gastro intestinal symptoms & 43 \\
\hline 2 & Giddiness & 14 \\
\hline 3 & Ocular toxicity & 0 \\
\hline 4 & Renal toxicity & 0 \\
\hline 5 & Arthralgia & 3 \\
\hline 6 & Cutaneous reactions & 3 \\
\hline 7 & Hepatitis & 1 \\
\hline 8 & Neurological symptoms & 1 \\
\hline 9 & Psychiatric disturbances & 1 \\
\hline 10 & Ototoxicity & 5 \\
\hline 11 & Hypothyroidism & 2 \\
\hline
\end{tabular}

Table .1 Frequency of adverse events. 
Impact Of Adverse Reactions On Adherence To Treatment For Mdr-Tb Under ....

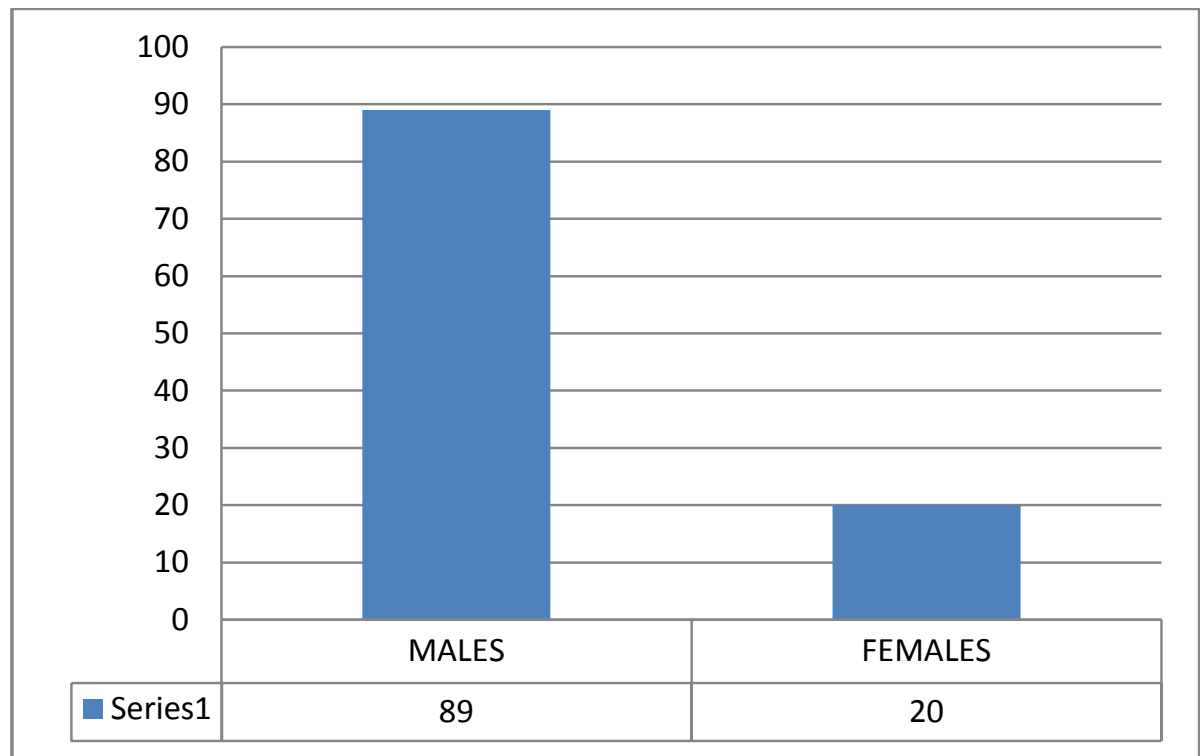

Fig.1 Gender distribution

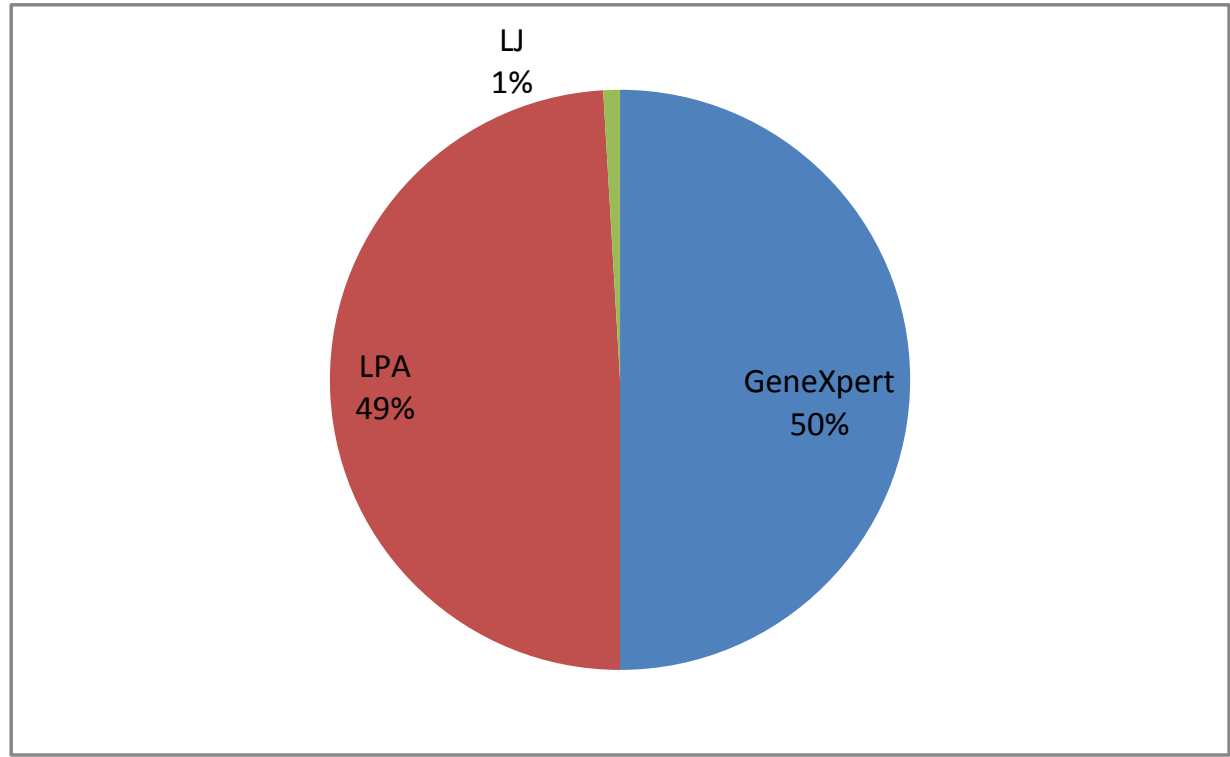

Fig.2 Drug sensitivity test

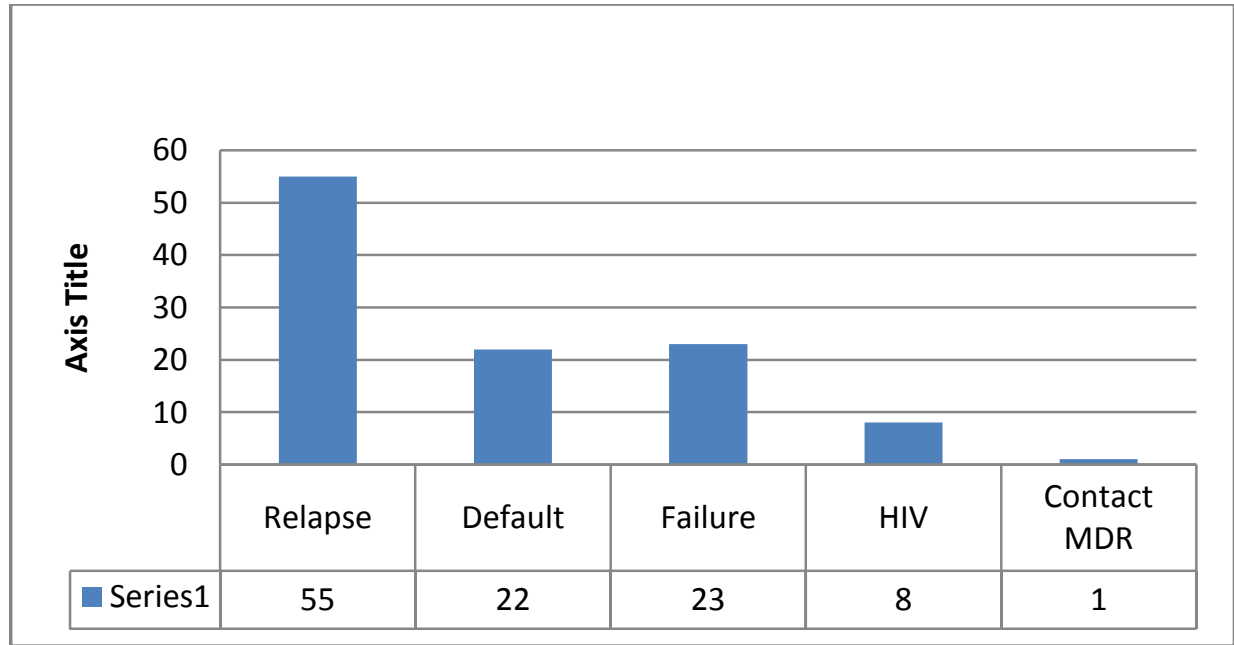

Fig.3 Indications for drug sensitivity testing 


\section{References}

[1]. Guidelines on Programmatic Management of Drug Resistant TB (PMDT) in India, May 2012

[2]. World Health Organization. Guidelines for establishing DOTSplus pilot projects for the management of MDR-TB. In: Gupta R, Arnadottir T, eds. WHO/CDS/TB/2000.279. Geneva, Switzerland:WHO, 2000.

[3]. Furin J J, Mitnick C D, Shin S S, et al. Occurrence of serious adverse events in patients receiving community based therapy for multidrug-resistant tuberculosis. Int J Tuberc Lung Dis 2001; 5: 648-655.

[4]. Goble M, Iseman M D, Madsen L A, Waite D, Ackerson L, Horsburgh C R Jr. Treatment of 171 patients with pulmonary tuberculosis resistant to isoniazid and rifampin. N Engl J Med 1993; 328: 527-532.

[5]. Yew W W, Chan $\mathrm{C} \mathrm{K}$, Chau $\mathrm{C} \mathrm{H}$, et al. Outcomes of patients with multidrug-resistant pulmonary tuberculosis treated with ofloxacin/ levofloxacin-containing regimens. Chest 2000; 117: 744-751.

[6]. Adverse events in the treatment of multidrug-resistant tuberculosis: results from the DOTS-Plus initiative E. Nathanson,* R.

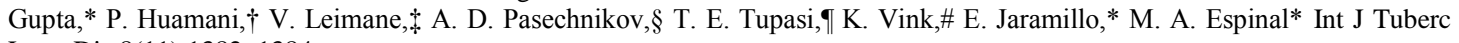
Lung Dis 8(11):1382-1384 